\title{
COVID-19: Taiwan's epidemiological characteristics and public and hospital responses
}

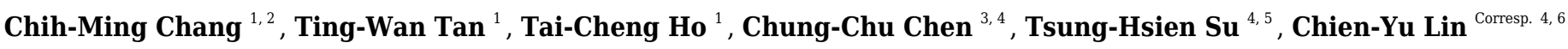 \\ 1 Department of Nursing, Hsinchu MacKay Memorial Hospital, Hsinchu, Taiwan \\ Graduate Institute of Health Industry Management, Yuanpei University, Hsinchu, Taiwan \\ 3 Department of Internal Medicine, Hsinchu MacKay Memorial Hospital, Hsinchu, Taiwan \\ 4 Department of Medicine, MacKay Medical College, New Taipei, Taiwan \\ 6 Department of Pediatrics, Hsinchu MacKay Memorial Hospital, Hsinchu, Taiwan \\ Corresponding Author: Chien-Yu Lin \\ Email address: mmhped.lin@gmail.com
}

Background: Coronavirus disease 19 (COVID-19) is a global health threat with significant medical, economic, social, and political implications. The optimal strategies for combating COVID-19 have not been fully determined and vary across countries.

Methods: By the end of February 2020 in Taiwan, 2,150 patients received diagnostic COVID-19 testing and 39 confirmed cases were detected. This is a relatively lower rate of infection compared to other Asian countries. In this article, we summarize the epidemiological characteristics of the 39 infected patients as well as public and hospital responses to COVID-19.

Results: Thirty-nine COVID-19 cases and one death have been confirmed in Taiwan. Seventeen of these patients were infected by family members or in hospital wards, emphasizing how COVID-19 is mostly spread by close contact. We examined how hospital have responded to COVID-19, including their implementation of patient route control, outdoor clinics, hospital visit restrictions, and ward and staff modifications. We also studied the public's use of face masks in response to COVID-19. These strategies may reduce the spread of COVID-19 in other countries.

Conclusion: The emergence and spread of COVID-19 is a threat to health worldwide. Taiwan has reported lower infected cases and its strategies may contribute to further disease prevention and control. 
2 COVID-19: Taiwan's epidemiological characteristics 3 and public and hospital responses

4

5 Chih-Ming Chang ${ }^{1}$, Ting-Wan Tan ${ }^{1}$, Tai-Cheng Ho ${ }^{1}$, Chung-Chu Chen ${ }^{2,}{ }^{3}$, Tsung-Hsien

$6 \mathrm{Su}^{3,4}$, Chien-Yu Lin ${ }^{3,5}$

7 'Department of Nursing, Hsinchu MacKay Memorial Hospital, Hsinchu,

8 Taiwan

9 'Department of Internal Medicine, Hsinchu MacKay Memorial Hospital, 10 Hsinchu, Taiwan

11 'Department of Medicine, MacKay Medical College, New Taipei, Taiwan

12 4Department of Obstetrics and Gynecology, Hsinchu MacKay Memorial

13 Hospital, Hsinchu, Taiwan

$14{ }^{5}$ Department of Pediatrics, Hsinchu MacKay Memorial Hospital, Hsinchu, 15 Taiwan

18 Corresponding Author:

19 Chien-Yu Lin

20 Hsinchu City, Taiwan

21 Email address: mmhped.lin@gmail.com 


\section{Abstract \\ Background:}

Coronavirus disease 19 (COVID-19) is a global health threat with

27

28 significant medical, economic, social, and political implications. The optimal strategies for combating COVID-19 have not been fully determined and vary across countries.

\section{Methods:}

By the end of February 2020 in Taiwan, 2,150 patients received diagnostic COVID-19 testing and 39 confirmed cases were detected. This is a relatively lower rate of infection compared to other Asian countries. In this article, we summarize the epidemiological characteristics of the 39 infected patients as well as public and hospital responses to COVID-19.

\section{Results:}

Thirty-nine COVID-19 cases and one death have been confirmed in Taiwan. Seventeen of these patients were infected by family members or in hospital wards, emphasizing how COVID-19 is mostly spread by close contact. We examined how hospital have responded to COVID-19, including their implementation of patient route control, outdoor clinics, hospital visit restrictions,-and ward and staff modifications. We also studied the public's use of face masks in response to COVID-19. These strategies may reduce the spread of COVID-19 in other countries.

\section{Conclusion:}


49 The emergence and spread of COVID-19 is a threat to health worldwide.

50 Taiwan has reported lower infected cases and its strategies may contribute 51 to further disease prevention and control.

52 
53

54

55

56

57

58

59

60

61

62

63

64

65

66

67

68

69

70

71

72

73

74

75

76

77

78

79

\section{Introduction}

The novel coronavirus, 2019-nCoV, emerged in Wuhan, China in December 2019 and has rapidly spread across the world (Gates 2020; Zhu et al. 2020). In February 2020, the World Health Organization (WHO) renamed this epidemic disease coronavirus disease (COVID-19) as it is caused by severe acute respiratory syndrome coronavirus 2 (SARS-CoV-2). The number of COVID-19 cases has rapidly increased worldwide ((WHO) 2020; del Rio \& Malani 2020). As of 29 February 2020, there were 85,403 confirmed COVID-19 cases and 2,924 fatalities across 49 countries ((WHO) 2020; Dong et al.). Although the emerging threat of COVID-19 has drawn global attention, the optimal strategies to reduce the spread of disease remain largely undetermined (Day 2020).

Several studies have investigated the virology, transmission, risk factors, and protection associated with COVID-19 (Chen et al. ; Guan et al. 2020; Huang et al. 2020; Wang et al. 2020b; Wu \& McGoogan 2020; Yang et al.). COVID-19 spreads by human-to-human transmission, primarily through respiratory droplets and direct contact with groups of infected family members, friends, colleagues, or medical health workers. However, even asymptomatic patients can be a source of infection. COVID-19 has dramatically higher rates of transmission compared to severe acute respiratory syndrome (SARS) and Middle East respiratory syndrome (MERS), but an observed lower fatality rate. The median age of COVID-19 patients ranged from 47 to 56 years old; approximately $65 \%$ of patients were male and $35 \%$ were female. Approximately $23.7 \%$ of adult COVID-19 patients have at least one of the following coexisting underlying chronic illnesses: cardiovascular disease, diabetes mellitus, hypertension, or chronic 
80 obstructive lung disease. The most common reported symptoms are fever 81 (43.8\% on admission and $88.7 \%$ during hospitalization), dry cough (67.8\%), 82 dyspnea (18.7\%), myalgia (14.9\%), headache (13.6\%), diarrhea $(3.8 \%)$, 83 sore throat $(13.9 \%)$, nasal obstruction $(4.8 \%)$, and fatigue $(38.1 \%)$. The 84 optimal infectious control measurements for COVID-19 have not yet been 85 established (Razai et al. 2020).

\section{Materials \& Methods}

\section{Study design and data sources}

We conducted this retrospective observational study at the end of February 2020 to investigate the epidemiological characteristics of the first 39 confirmed COVID-19 cases in Taiwan. The Taiwan Centers for Disease 
106 Control (TCDC) enforced many policies to combat the spread of COVID-19 107 (Centers for Disease Control 2020). The epidemiological data of the 108 confirmed cases were released to the public. Although the identifiable data 109 were encrypted, the clinical presentations and epidemiological relationships 110 were reported, and data regarding demographics, clinical symptoms, contact 111 history, and onset dates were extracted. We summarized the public data to 112 show the clinical characteristics of the 39 cases.

2. COVID-19 diagnoses

Since COVID-19 is considered a communicable disease in Taiwan, all suspected cases are required to be reported to the TCDC. The clinical COVID-19 diagnostic criteria include a history of travel or residence in endemic areas, contact with confirmed cases, contact with cases of fever or respiratory symptoms, radiological image characteristics of pneumonia, and clinical manifestations of fever or respiratory tract symptoms. Early diagnosis and timely containment are important. The TCDC's diagnostic testing criterion included: (1) patients from endemic areas with respiratory symptoms; (2) patients with unexplained pneumonia or community pneumonia and poor responses to antibiotic treatment; (3) patients in close contact with confirmed cases; and (4) cluster infections or unwell people in specific workplaces, such as hospitals or nurseries. The defined endemic areas varied over time.

Suspected cases were confirmed by nucleic acid detection from the sputum, throat swab, lower respiratory tract secretion, or blood samples. The polymerase chain reaction for COVID-19 and other viruses were performed (Cui et al. 2019; Lin et al. 2020; Lu et al. 2020b; The 2020). The suspected 
132 cases were isolated in negative pressure rooms or single bedrooms until a 133 second negative test result.

\section{COVID-19 treatment}

Since there is no specific treatment for COVID-19, the main treatments used are supportive care and oxygen support ((WHO) 2020; Centers for Disease Control 2020). Suspected or confirmed cases were moved to isolated rooms and received supportive treatment to ensure electrolyte balance, closely monitored vital signs, and oxygen saturation. Routine blood tests and arterial blood gas analyses were administered if necessary. Chest imaging and other procedures were arranged. Patients with pneumonia received intravenous broad-spectrum antibiotics such as penicillin or 144 ceftriaxone, and oseltamivir was also administered empirically. For confirmed cases, they could leave isolation and return home after three consecutive negative PCR tests. In average, it took approximately one month to achieve the criteria of de-isolation thus the capacity of isolated rooms was an important issue.

\section{Public health and hospital responses}

Widespread anxiety is anticipated when facing a novel and unknown 152 infection (The 2020). The TCDC executed several policies, but individual 153 hospitals were authorized to implement their own infective control 154 measurements against COVID-19 in addition to their normal practices. For example, decreasing exposure was important to reduce disease spread, and

156 hospitalized patients are susceptible to various kinds of infectious diseases 157 including COVID-19. To reduce viral transmission, hospitals set up outdoor 
158 clinics to care for patients with travel or contact histories. We investigated 159 other important health polices and hospital responses.

160

161

162

163

164

165

166

167

168

169

170

171

172

173

174

175

176

177

178

179

180

181

182

183

184

\section{Results}

1. Epidemiological characteristics of confirmed cases

We summarized cumulative cases from other Asian countries starting in mid-February (Figure 1). By the end of February 2020, 2,150 patients in Taiwan had been tested for COVID-19. Among them, 39 (1.8\%) patients tested positive for SARS-CoV-2. The epidemiological characteristics of the 39 confirmed cases are summarized in Table 1. Sixteen (41.0\%) patients were male and $23(59.0 \%)$ were female. Approximately half (53.8\%) of these patients were between 41 and 60 years old. Nineteen cases (48.7\%) had a history of overseas travel or residence or had visited China. Fifteen (38.5\%) of the confirmed cases had direct contact transmission from infected family members. One patient $(2.6 \%)$ died. The TCDC aggressively tracked the travel, occupation, contact, and cluster histories of the confirmed cases. The correlations among the 39 cases were plotted and publicly released (Figure 2).

2. Public health and hospital responses to COVID-19

2.1. Home quarantine and patient route control

Hospitals designated separate entrances and exits for patients and the public to prevent hospital-acquired COVID-19 infections. Suspected cases were treated separately from the emergency and outpatient cases throughout the duration of their hospital stay. When scheduling appointments online, the Tracking and Management Mechanism for People under Infection 
185 Risk announcement appeared (Figure 3). If patients had any history of travel 186 to endemic areas or of respiratory infection, they were guided to a special 187 COVID-19 outdoor clinic (Figure 4).

188 Suspected cases were guided to an outdoor triage and then admitted to 189 an isolation room. Diagnostic tests were performed in negative pressure 190 rooms or single bedrooms. Hand sanitizer, masks, and COVID-19-related

191

192

193

194

195

196

197

198

199

200

201

202

203

204

205

206

207

208

209

210

211 posters were posted at the hospital entrances. Patients and visitors had to wash their hands with alcohol hand gel before entering the hospital. These infectious control measurements all contributed to reducing hospitalacquired infections.

\subsection{Hospital visit restrictions}

Hospital visits are common in Asian cultures, so hospitals in Taiwan restricted visits to prevent COVID-19 infections and outbreaks. Instead of face-to-face hospital visits, video calls were recommended for family and friends to contact patients. Ward entrances were controlled by electromagnetic doors requiring access cards. Patients were not allowed in restricted areas. No more than two visitors were allowed to visit the same patient at the same time. Visitors with recent travel histories were restricted from entering the hospital (Figure 5). In the case of a confirmed infection, visitor registration provided clues for tracking the infectious source and their contacts.

\subsection{Regulation of masks and other personal protective equipment}

Surgical masks are basic protections that prevent human-to-human transmission of COVID-19. Taiwanese people tend to wear masks in large and crowded gathering areas in their daily lives to protect themselves and 
212 others. In order to prevent public over-purchase and hoarding, the 213 Taiwanese government took actions to recruit the mask factories and 214 allocate all masks. The government helped factories upgrade their facilities, 215 and mask production increased from 1 million per day in early February to 21610 million per day in late February. The Taiwanese government issued 217 surgical masks to all local hospitals and clinics to prevent shortages. Each 218 medical staff member received one to two masks every day for regular use, 219 and additional masks or N95 masks were provided for healthcare providers 220 taking care of COVID-19 patients or performing invasive procedures. 221 Moreover, the government provided surgical masks for susceptible 222 populations, such as patients with cancer, hemodialysis, or those undergoing 223 chemotherapy and radiotherapy. Upon hospital admission, one mask was 224 given to patients with reduced nosocomial infection or hospital-acquired 225 infection. To reduce community transmission, the government also issued 226 surgical masks to children. Taiwanese residents needed to present a 227 national health insurance card to purchase surgical masks from local 228 pharmacies and primary healthcare units. This policy allowed each 229 residence to purchase two masks weekly and prohibited repeat purchases.

\subsection{Ward and staff modifications}

Suspected and confirmed cases were admitted to their own rooms to 233 ensure isolation. In order to minimize infections of healthcare providers, nursing staff adhered to group scheduling and fixed shifting, separated by 235 meal breaks. Job and ward rotations were prohibited to reduce the chances of hospital-acquired transmission. 


\section{Discussion}

240

COVID-19 is a global health threat, but Taiwan has a relatively lower rate

of infection compared to other countries. Approximately $50 \%$ of Taiwan's 39 confirmed cases were imported and $38.5 \%$ were infected by their own families. Aggressive tracking and epidemiological surveillance strategies contributed to identifying infectious sources and reducing disease spread. Furthermore, infectious control measurements in hospitals may have reduced the transmission of COVID-19.

There are no established best strategies against COVID-19 and policies differ across populations, geographic regions, cultures, countries, and time. "Lockdown" was a powerful strategy used by China to isolate infected people 252 and reduce transmission, but it is not easily replicated in many countries. The 253 United Kingdom's "herd immunity" strategy also caught our attention (YONG 254 2020). However, Taiwan's close proximity to and frequent interactions with China suggest that following the UK's strategy could result in the collapse of 256 the medical system. Moreover, convenience in travelling contributed to virus transmission thus disease spread was almost inevitable. The rapid spread 258 of COVID-19 had been observed in UK and the "herd immunity" strategy had 259 been abandoned. The UK government made efforts towards "containment" 260 to slow down the virus transmission.(Boyle 2020) We were still exploring the appropriate strategies to tackle COVID-19 in different situations.

Aggressive control measurements in Taiwan helped flatten the infectious 263 curve, prevent medical collapse, and reduce case fatality rates. For example, 264 there were 1,300 isolation rooms with an occupancy rate of approximately 
$26570 \%$ in February 2020 (Centers for Disease Control 2020). Adequate 266 isolation rooms ensured adequate containment and reduced the risk of 267 community infections. Furthermore, wearing masks is already prevalent in 268 Taiwanese society, and masks provide simple and direct protection against 269 droplet infection (Leung et al. 2020). Wearing masks has not been a common 270 practice in other countries (Desai \& Mehrotra 2020). Taiwan's aggressive 271 strategies ensured the provision of adequate masks, decreasing both anxiety 272 among the public and disease spread.

273 Although, the culture and population density of Japan are relatively similar 274 to Taiwan's, Japan's situation was more complicated. They faced the 275 complex issues with the Diamond Princess cruise ship carrying infected 276 patients, quarantined in the port of Yokohama, Japan, and the Olympics, and 277 they used different strategies to combat COVID-19 (Gallego et al. 2020; 278 Montanari 2020). Optimal strategies varied across different countries and 279 changed over time.

280

The COVID-19 pandemic is a rapidly changing situation. According to 282 a study by the Chinese CDC, the majority of infected people are aged 30 to 283 79 years (87\%). In Taiwan's 39 confirmed cases, 35 (90\%) were aged $30-79$ 284 years. Taiwan's case fatality rate $(1 / 39,2.56 \%)$ was similar to China's 285 (1023/44672, 2.3\%) (Wu \& McGoogan 2020). Approximately half of 286 Taiwanese patients $(19 / 39,48.7 \%)$ had an overseas travel history, but the 287 high risk of community infection should not be ignored. Family and ward 288 clusters are important transmission sources, and 22 people across five 289 families were identified in the 39 reported cases. The high contagiousness 290 of SARS-CoV-2 makes disease prevention difficult. Moreover, $81 \%$ of 291 infected patients reported mild symptoms which were easily overlooked 
292 leading to delayed diagnoses (Bai et al. 2020; Rothe et al. 2020). Aggressive 293 tracking and infectious source identification lead to early quarantine, 294 transmission prevention, and early diagnoses of patients with mild 295 symptoms.

296

Although SARS-CoV-2's complete route of transmission has not been 298 completely identified, it is mainly spread via droplets and physical contact ( $\mathrm{Li}$ 299 et al. 2020; Lu et al. 2020a; Xu et al. 2020). Wearing masks and washing 300 hands are essential and effective in preventing the spread of infectious 301 disease (Leung et al. ; Maclntyre \& Chughtai 2015). Public panic is expected 302 when an emerging disease appears likely to become endemic or a 303 widespread epidemic (Bao et al. 2020; Medley \& Vassall 2017), leading to a 304 shortage of masks and increased risk of disease transmission. The TCDC's 305 mask policy is an executable and effective strategy to combat disease. 306 Furthermore, hospitals implementing visitation restrictions and outdoor 307 clinics may also reduce the risk of COVID-19 transmission.

Technological advances also contribute to improvements in infection 310 control. Big data analytics, new technology, and proactive testing have been applied in the war against COVID-19 (Wang et al. 2020a). A real-time, 312 interactive internet dashboard has also been utilized to provide timely 313 information for the general public and healthcare providers (Dong et al.). 314 These strategies are believed to be beneficial in preventing and controlling 315 disease spread. We summarized some additional hospital responses and 316 provided photographs for healthcare providers' reference (Figures 1, 3, 4, 5). 
Our study had some limitations. First, COVID-19 is a communicable 319 disease and universal screening is not currently available. The number of 320 patients with mild symptoms may be underestimated. Second, since SARS321 CoV-2's virology, transmission, incubation period, and contagious period are 322 not fully understood, the most effective strategies to use against COVID-19 323 remain speculative. More studies are required to investigate the 324 effectiveness of individual infectious control measurements.

\section{Conclusions}

327 The emergence of COVID-19 is a critical global issue. Taiwan's 39 328 confirmed cases showed a similar age distribution as previous studies (Guan 329 et al., 2020). The role of family and ward clusters in disease transmission 330 was emphasized. Public health and hospitals' patient route control, outdoor 331 clinics, hospital visit restrictions, mask regulations, and ward and staff 332 modification strategies may contribute to reducing the transmission of 333 COVID-19.

334

335

336

337

338

339

340

341

342

343

344

345

346

347

\section{Acknowledgements}

We thank the Centers for Disease Control, Taiwan and all efforts to combat COVID-19.

We thank David Singleton for his brilliant review and constructive comments. The quality of our manuscript has been improved following his precious suggestions.

\section{References}


348

349

350

351

352

353

354

355

356

357

358

359

360

361

362

363

364

365

366

367

368

369

370

371

372

373

374

375

376

377

378

379

380

381

382

383

384

385

386

387

388

389

390

391

392

393

394

395

396

397

(WHO) WHO. 2020. Novel coronavirus (2019-nCoV). Situation report. Available at https://www.who.int/emergencies/diseases/novel-coronavirus-2019/situation-reports (accessed 14March 2020).

Bai Y, Yao L, Wei T, Tian F, Jin D-Y, Chen L, and Wang M. 2020. Presumed Asymptomatic Carrier Transmission of COVID-19. JAMA. 10.1001/jama.2020.2565

Bao Y, Sun Y, Meng S, Shi J, and Lu L. 2020. 2019-nCoV epidemic: address mental health care to empower society. The Lancet 395:e37-e38. 10.1016/S0140-6736(20)30309-3

Boyle C. 2020. On coronavirus containment, Britain's Johnson is less restrictive than other European leaders. Available at https://www.latimes.com/world-nation/story/2020-0319/british-prime-minister-boris-johnsons-coronavirus-european-leaders (accessed 05May2020.

Centers for Disease Control T. 2020. Coronavirus disease-19. Available at https://www.cdc.gov.tw/En (accessed 14March 2020).

Chen H, Guo J, Wang C, Luo F, Yu X, Zhang W, Li J, Zhao D, Xu D, Gong Q, Liao J, Yang H, Hou W, and Zhang Y. Clinical characteristics and intrauterine vertical transmission potential of COVID-19 infection in nine pregnant women: a retrospective review of medical records. The Lancet. 10.1016/S0140-6736(20)30360-3

Chen X, Tian J, Li G, and Li G. Initiation of a new infection control system for the COVID-19 outbreak. The Lancet Infectious Diseases. 10.1016/S1473-3099(20)30110-9

Cui J, Li F, and Shi ZL. 2019. Origin and evolution of pathogenic coronaviruses. Nat Rev Microbiol 17:181-192. 10.1038/s41579-018-0118-9

Day M. 2020. Covid-19: surge in cases in Italy and South Korea makes pandemic look more likely. BMJ 368:m751. 10.1136/bmj.m751

del Rio C, and Malani PN. 2020. COVID-19-New Insights on a Rapidly Changing Epidemic. JAMA. 10.1001/jama.2020.3072

Desai AN, and Mehrotra P. 2020. Medical Masks. JAMA. 10.1001/jama.2020.2331

Dong E, Du H, and Gardner L. An interactive web-based dashboard to track COVID-19 in real time. The Lancet Infectious Diseases. 10.1016/S1473-3099(20)30120-1

Gallego V, Nishiura H, Sah R, and Rodriguez-Morales AJ. 2020. The COVID-19 outbreak and implications for the Tokyo 2020 Summer Olympic Games. Travel Med Infect Dis:101604. 10.1016/j.tmaid.2020.101604

Gates B. 2020. Responding to Covid-19 - A Once-in-a-Century Pandemic? New England Journal of Medicine. 10.1056/NEJMp2003762

Guan W-j, Ni Z-y, Hu Y, Liang W-h, Ou C-q, He J-x, Liu L, Shan H, Lei C-I, Hui DSC, Du B, Li Lj, Zeng G, Yuen K-Y, Chen R-c, Tang C-I, Wang T, Chen P-y, Xiang J, Li S-y, Wang J-I, Liang Z-j, Peng Y-x, Wei L, Liu Y, Hu Y-h, Peng P, Wang J-m, Liu J-y, Chen Z, Li G, Zheng Z-j, Qiu S-q, Luo J, Ye C-j, Zhu S-y, and Zhong N-s. 2020. Clinical Characteristics of Coronavirus Disease 2019 in China. New England Journal of Medicine. 10.1056/NEJMoa2002032

Huang C, Wang Y, Li X, Ren L, Zhao J, Hu Y, Zhang L, Fan G, Xu J, Gu X, Cheng Z, Yu T, Xia J, Wei Y, Wu W, Xie X, Yin W, Li H, Liu M, Xiao Y, Gao H, Guo L, Xie J, Wang G, Jiang R, Gao Z, Jin Q, Wang J, and Cao B. 2020. Clinical features of patients infected with 2019 novel coronavirus in Wuhan, China. The Lancet 395:497-506. 10.1016/S01406736(20)30183-5

Leung CC, Lam TH, and Cheng KK. Mass masking in the COVID-19 epidemic: people need guidance. The Lancet. 10.1016/S0140-6736(20)30520-1

Leung NHL, Chu DKW, Shiu EYC, Chan K-H, McDevitt JJ, Hau BJP, Yen H-L, Li Y, Ip DKM, Peiris JSM, Seto W-H, Leung GM, Milton DK, and Cowling BJ. 2020. Respiratory virus shedding in exhaled breath and efficacy of face masks. Nature Medicine. 10.1038/s41591-020-0843-2

Peer) reviewing PDF | (2020:03:46677:2:0:CHECK 5 May 2020) 
398

399

400

401

402

403

404

405

406

407

408

409

410

411

412

413

414

415

416

417

418

419

420

421

422

423

424

425

426

427

428

429

430

431

432

433

434

435

436

437

438

439

440

441

442

443

444

445

446

447

448

Li Q, Guan X, Wu P, Wang X, Zhou L, Tong Y, Ren R, Leung KSM, Lau EHY, Wong JY, Xing X, Xiang N, Wu Y, Li C, Chen Q, Li D, Liu T, Zhao J, Liu M, Tu W, Chen C, Jin L, Yang R, Wang Q, Zhou S, Wang R, Liu H, Luo Y, Liu Y, Shao G, Li H, Tao Z, Yang Y, Deng Z, Liu B, Ma Z, Zhang Y, Shi G, Lam TTY, Wu JT, Gao GF, Cowling BJ, Yang B, Leung GM, and Feng Z. 2020. Early Transmission Dynamics in Wuhan, China, of Novel Coronavirus-Infected Pneumonia. New England Journal of Medicine. 10.1056/NEJMoa2001316

Lin C-Y, Hwang D, Chiu N-C, Weng L-C, Liu H-F, Mu J-J, Liu C-P, and Chi H. 2020. Increased Detection of Viruses in Children with Respiratory Tract Infection Using PCR. International Journal of Environmental Research and Public Health 17:564.

Lu C-w, Liu X-f, and Jia Z-f. 2020a. 2019-nCoV transmission through the ocular surface must not be ignored. The Lancet 395:e39. 10.1016/S0140-6736(20)30313-5

Lu R, Zhao X, Li J, Niu P, Yang B, Wu H, Wang W, Song H, Huang B, Zhu N, Bi Y, Ma X, Zhan F, Wang L, Hu T, Zhou H, Hu Z, Zhou W, Zhao L, Chen J, Meng Y, Wang J, Lin Y, Yuan J, Xie Z, Ma J, Liu WJ, Wang D, Xu W, Holmes EC, Gao GF, Wu G, Chen W, Shi W, and Tan W. 2020b. Genomic characterisation and epidemiology of 2019 novel coronavirus: implications for virus origins and receptor binding. The Lancet 395:565-574. 10.1016/S0140-6736(20)30251-8

Maclntyre CR, and Chughtai AA. 2015. Facemasks for the prevention of infection in healthcare and community settings. BMJ : British Medical Journal 350:h694. 10.1136/bmj.h694

Medley GF, and Vassall A. 2017. When an emerging disease becomes endemic. Science 357:156-158. 10.1126/science.aam8333

Montanari S. 2020. Japan has a remarkably low number of coronavirus cases that experts worry may lead to a 'false sense of security'. Available at https://www.businessinsider.com/why-japan-cases-of-coronavirus-are-so-low-2020-3 (accessed 03/20 2020).

Razai MS, Doerholt K, Ladhani S, and Oakeshott P. 2020. Coronavirus disease 2019 (covid19): a guide for UK GPs. BMJ 368:m800. 10.1136/bmj.m800

Rothe C, Schunk M, Sothmann P, Bretzel G, Froeschl G, Wallrauch C, Zimmer T, Thiel V, Janke C, Guggemos W, Seilmaier M, Drosten C, Vollmar P, Zwirglmaier K, Zange S, Wölfel R, and Hoelscher M. 2020. Transmission of 2019-nCoV Infection from an Asymptomatic Contact in Germany. New England Journal of Medicine. 10.1056/NEJMc2001468

The L. 2020. COVID-19: fighting panic with information. The Lancet 395:537. 10.1016/S01406736(20)30379-2

Wang CJ, Ng CY, and Brook RH. 2020a. Response to COVID-19 in Taiwan: Big Data Analytics, New Technology, and Proactive Testing. JAMA. 10.1001/jama.2020.3151

Wang D, Hu B, Hu C, Zhu F, Liu X, Zhang J, Wang B, Xiang H, Cheng Z, Xiong Y, Zhao Y, Li Y, Wang X, and Peng Z. 2020b. Clinical Characteristics of 138 Hospitalized Patients With 2019 Novel Coronavirus-Infected Pneumonia in Wuhan, China. JAMA. 10.1001/jama.2020.1585

Wu Z, and McGoogan JM. 2020. Characteristics of and Important Lessons From the Coronavirus Disease 2019 (COVID-19) Outbreak in China: Summary of a Report of 72314 Cases From the Chinese Center for Disease Control and Prevention. JAMA. 10.1001/jama.2020.2648

Xu X-W, Wu X-X, Jiang X-G, Xu K-J, Ying L-J, Ma C-L, Li S-B, Wang H-Y, Zhang S, Gao H-N, Sheng J-F, Cai H-L, Qu Y-Q, and Li L-J. 2020. Clinical findings in a group of patients infected with the 2019 novel coronavirus (SARS-Cov-2) outside of Wuhan, China: retrospective case series. BMJ 368:m606. 10.1136/bmj.m606

Yang X, Yu Y, Xu J, Shu H, Xia Ja, Liu H, Wu Y, Zhang L, Yu Z, Fang M, Yu T, Wang Y, Pan S, Zou $X$, Yuan S, and Shang Y. Clinical course and outcomes of critically ill patients with

Peer) reviewing PDF | (2020:03:46677:2:0:CHECK 5 May 2020) 
SARS-CoV-2 pneumonia in Wuhan, China: a single-centered, retrospective, observational study. The Lancet Respiratory Medicine. 10.1016/S2213-2600(20)30079-5 https://www.theatlantic.com/health/archive/2020/03/coronavirus-pandemic-herdimmunity-uk-boris-johnson/608065/ (accessed 2020/03/16.

Zhu N, Zhang D, Wang W, Li X, Yang B, Song J, Zhao X, Huang B, Shi W, Lu R, Niu P, Zhan F, Ma X, Wang D, Xu W, Wu G, Gao GF, and Tan W. 2020. A Novel Coronavirus from Patients with Pneumonia in China, 2019. New England Journal of Medicine 382:727-

458 733. 10.1056/NEJMoa2001017 
Figure 1

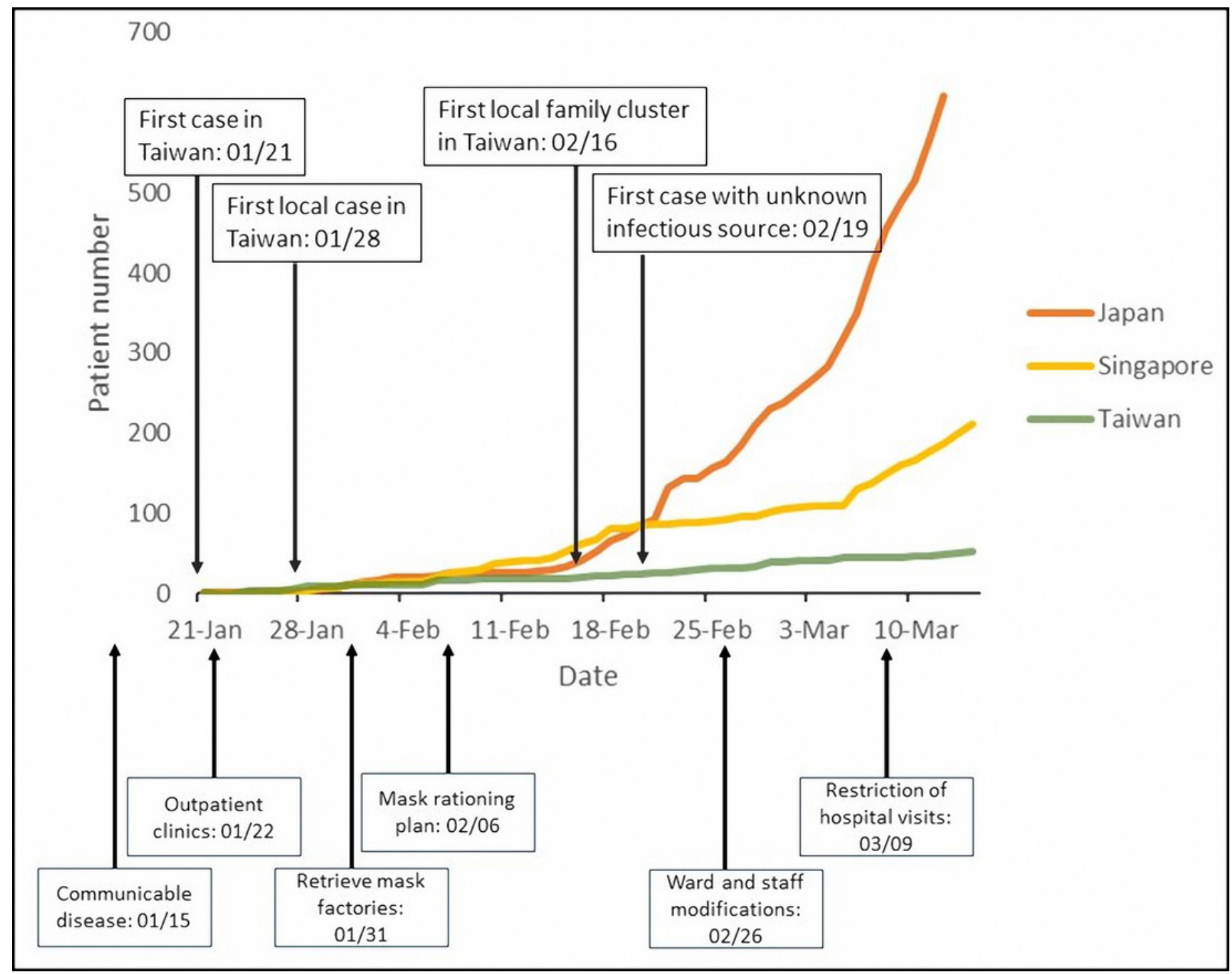


Figure 2 


\section{Relationship between confirmed cases}

Produced by Central

Epidemic Command

Center, 03 March

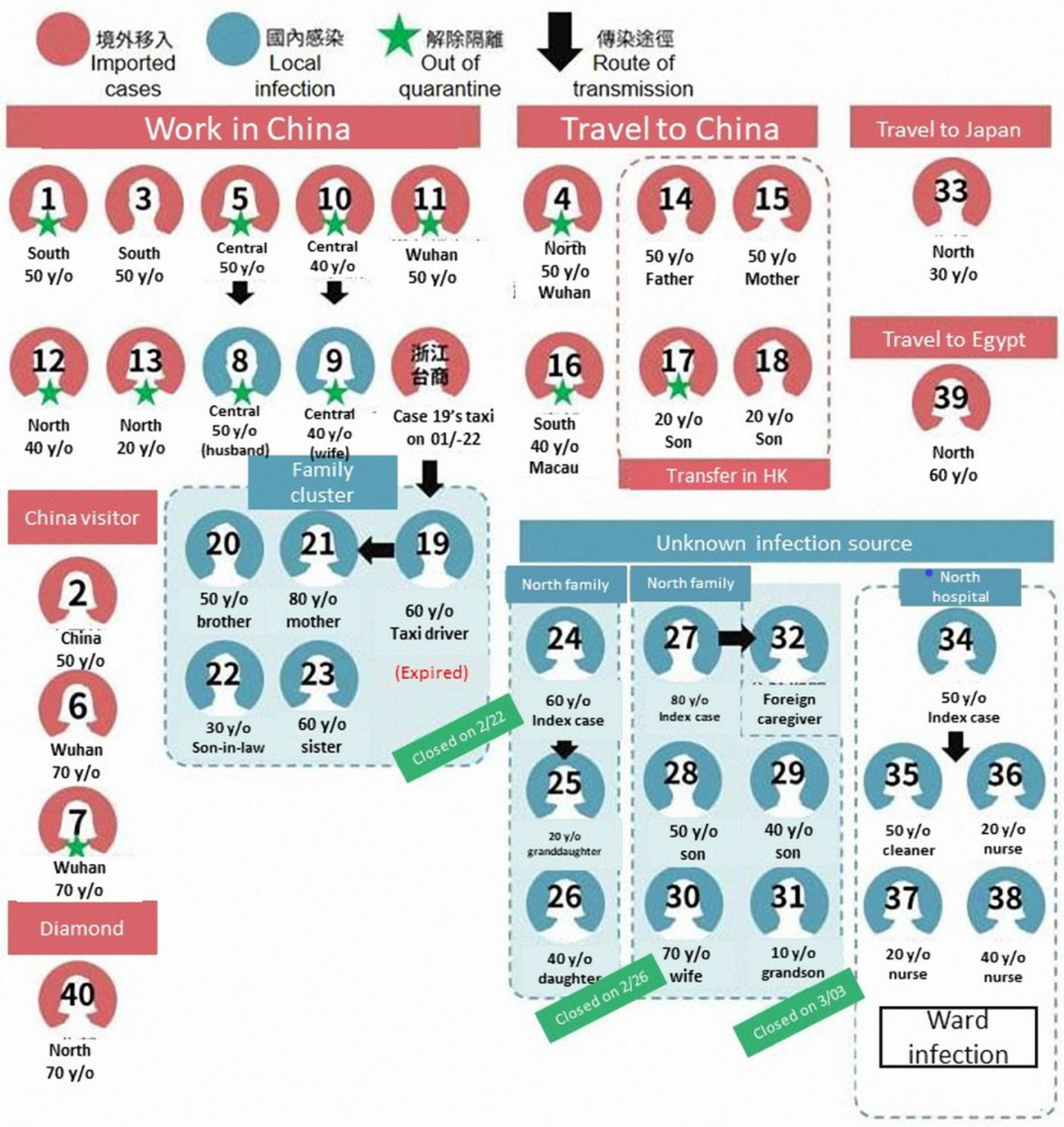

*Adopted and modified by Taiwan Centers for Disease Control. Publication under the terms and conditions of the Creative Commons Attribution (CC BY) license

*Dotted lines: family cluster or ward cluster; pink color: imported cases; blue color: local infection; green star: out of quarantine; black arrow: route of transmission 
Figure 3

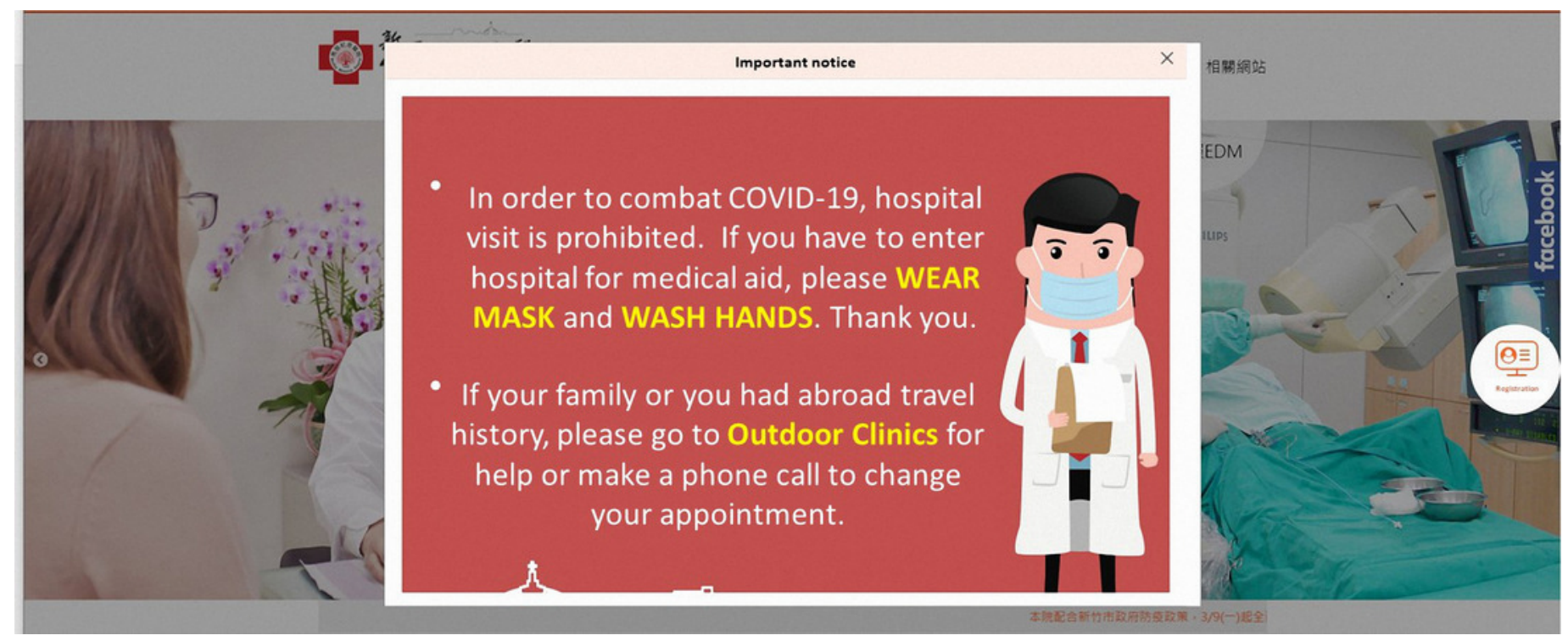


Figure 4

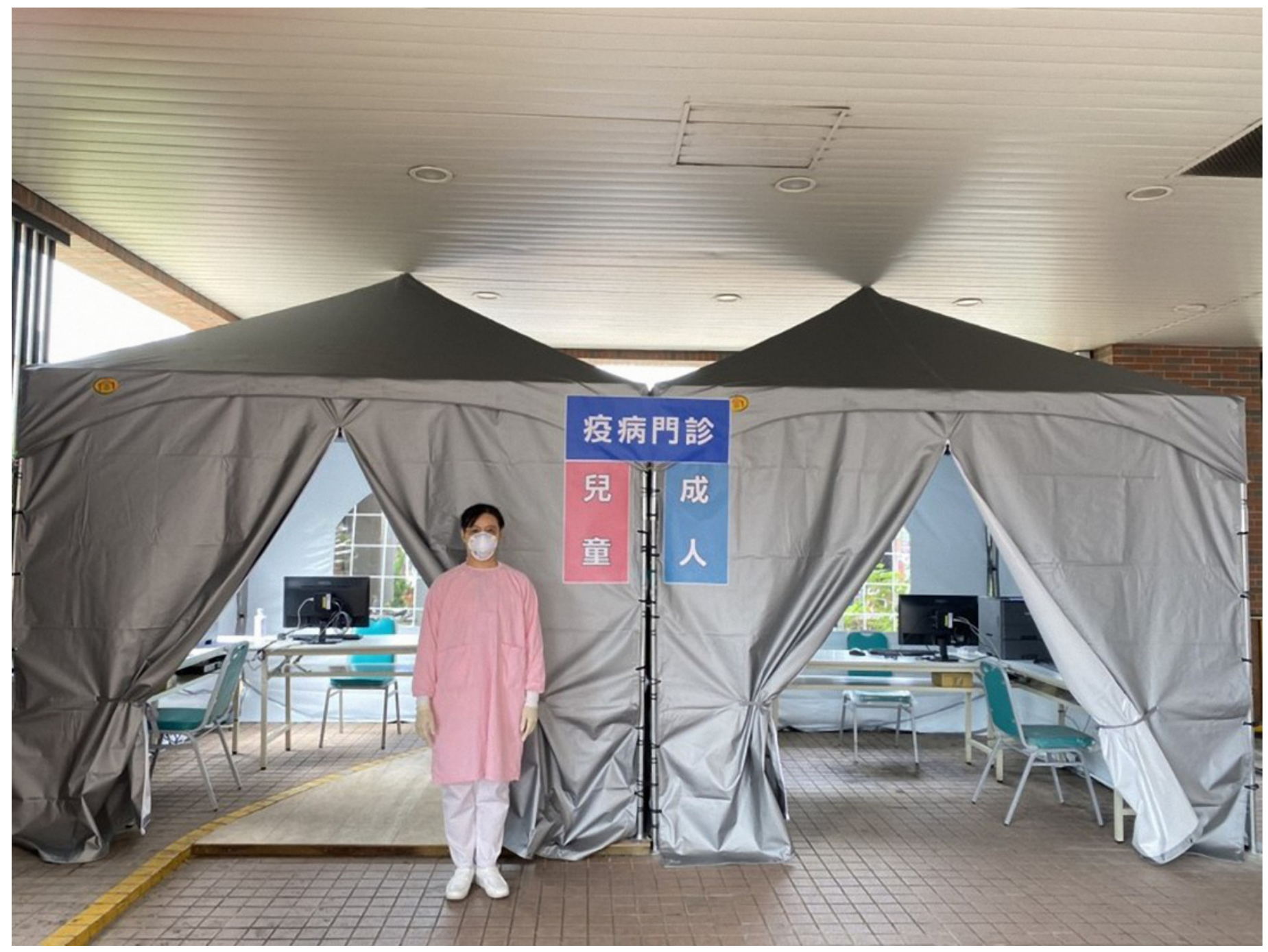


Figure 5

A shorter version of visitor restriction was posted to provide clear instructions to patients and visitors. If visitors were allowed to enter hospital, registration with detailed information was required for further contact tracing.

\section{To combat COVID-19 and protect yourself,}

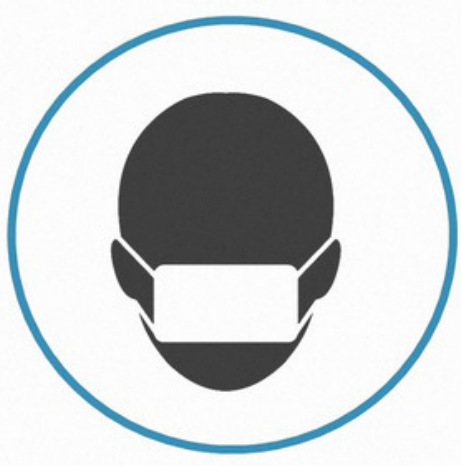

Wearing mask

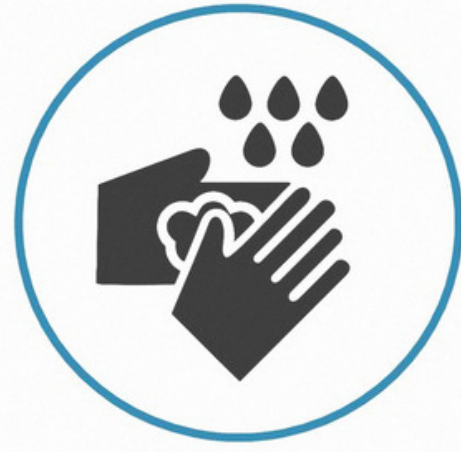

Hand washing

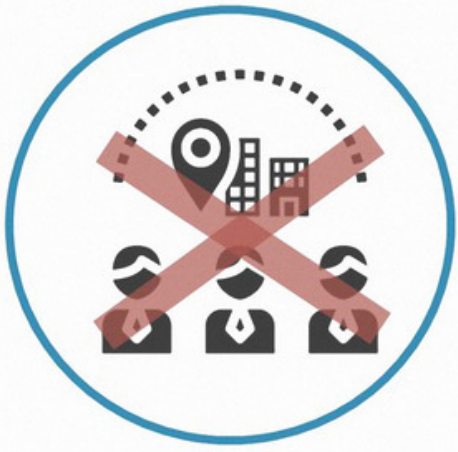

Restriction of hospital visits

Hsinchu MacKay Memorial Hospital: (03)6119595-2295 
Table $\mathbf{1}$ (on next page) 
1

\begin{tabular}{cccc}
\hline Clinical characteristics & & No. & Percentage (\%) \\
\hline Total & male & 39 & 100 \\
\hline Gender & female & 18 & 53.8 \\
& $11 \sim 20$ & 2 & 46.2 \\
\hline Age (years old) & $21 \sim 30$ & 4 & 5.1 \\
& $31 \sim 40$ & 4 & 10.3 \\
& $41 \sim 50$ & 10 & 10.3 \\
& $51 \sim 60$ & 11 & 25.6 \\
& $61 \sim 70$ & 3 & 28.2 \\
& $71 \sim 80$ & 3 & 7.7 \\
& $>81$ & 2 & 7.7 \\
& none & 20 & 5.1 \\
\hline Travel history & yes & 19 & 51.3 \\
& none & 21 & 48.7 \\
\hline Family cluster infection & yes & 17 & 53.8 \\
& unknown source & 1 & 43.6 \\
& & & 2.6 \\
\hline
\end{tabular}

2

3 